\title{
The role of endocrine disruptors in the present and future human endocrine evolution: The ed-exohormone-system
}

\author{
Csaba $G^{*}$ \\ Department of Genetics, Cell- and Immunobiology, Semmelweis University, Budapest, Hungary
}

\begin{abstract}
In this paper a theory on the possible transformation of endocrine disruptors (EDs) to real hormones, is introduced. The evidences, demonstrating the possibility of transformation, are reviewed. Lipid soluble vitamins (in fact, exo-hormones, e.g. vitamins A and D)), which once could have also acted as endocrine disruptors, were converted to physiologically active hormones during evolution, when they meet a claim. Our model yields to the prediction that a similar conversion will happen by phytoestrogens. These already have a role in human food consumption since thousand years having both beneficial and harmful effects. However, the conversion of other (e.g. industrial) disruptors (the number and amount of which are enormously growing) is also likely. The (non-mutational) epigenetic inheritance of faulty hormonal imprinting helps the stabilization of evolutionary changes. From this aspect the effect of EDs can also be useful, not only harmful, as it is believed up to now. The apparently harmful effects of endocrine disruptors (e.g. on male fertility) may also be beneficial in a certain respect, considering human overpopulation and its consequences. At present the whole human endocrine system is attacked by environmental and medical endocrine disruptors, causing the imbalance of the system, creating conditions of epigenetically inheriting changes. The goal of this paper to emphasize the possibility of evolutionary changes caused by the ubiquity of endocrine disruptors and to their importance in our present and future life.
\end{abstract}

\section{Introduction}

\section{Background}

The human evolution has not stopped [1] and new kind of factors are shaping it. Some of these factors originate from human activity itself and they are often followed by a backlash manifested in evolutionary alterations. This means that the human evolution of the present age (and the future) is a consequence of the general conveyed evolution [2] of mankind (the transfer of human abilities to tools).

The hormonal regulation and control of mammalian organisms is one of the most important factors besides the nervous system. It is genetically controlled and under normal circumstances it is well balanced. However, human chemical interventions, which contaminate the environment can disturb it. The chemical components participating in this process are called endocrine disruptors (EDs), substances which are products of the industry (e.g. bisphenol A, the most widespread ED which is used in plastic industry and bound by estrogen receptors; vinclozolin, which is used in the agrotechnics and bound by the androgen receptors etc), the communal tools (e.g. benzpyrene, dioxin), or plant products (e.g. soy hormone genistein, bound by estrogen receptors) used in the feeding human.The EDs can be bound to (intracellular) hormone receptors, acutely activating or inhibiting the response of the receptor-bearing cell [3]. However, in critical phases of receptor development (prenatally or during adolescence), they may provoke faulty hormonal imprinting, influencing binding capacity of receptors for life [4-6].

Hormonal imprinting is a physiological process, which is taking place perinatally during the first encounter between the hormone and its target cell and it is absolutely needed for the normal receptor-hormone binding in adulthood. This process is disturbed by the presence (excess) of strange ED-s, which can be bound by the developing receptor.
This faulty imprinting by hormone-like materials alters the normal imprinting.

Most of the ED-s are steroid-like molecules, or such molecules which can be bound by steroid hormone receptors. This effect is inherited epigenetically [7] to the progeny generations. Considering facts, the EDs can intervene in the human evolution by inducing faulty steroid hormone receptor formation and provoking routes for the transmission of receptor-perceived information [8].

\section{Lipid -soluble vitamins as models for the evolutionary role of endocrine disruptors}

The ubiquitousness of EDs is characteristic to our present age, despite the fact that EDs were present in the human environment earlier, and could possibly had an evolutionary role.The substances now called lipid-soluble vitamins, such as vitamins A, D, E and K (which in fact hormones, as they are bound by hormone receptors, inducing response) could have been such EDs in earlier times. Vitamin A is (and was) never synthesized in the human body, however this lipidsoluble vitamin can be found in many plants, which synthesized it or its provitamin, beta carotene. If vitamin A was introduced into the human body, it found receptors among the steroid receptor superfamily which were also able to bind it. If the response was beneficial for

${ }^{*}$ Correspondence to: Gyorgy Csaba MD, PhD, DSc, Professor Emeritus, Department of Genetics, Cell- and Immunobiology, Semmelweis University, Budapest, Hungary, E-mail: csaba.gyorgy@med.semmelweis-univ.hu

Key words: evolution, endocrine system, lipid soluble vitamins, hormones, hormonal imprinting, vitamin $A$, vitamin $D$

Received: January 07, 2019; Accepted: January 29, 2019; Published: January 31, 2019 
the organism, a special receptor could have developed and fixed for specifically binding the vitamin (retinoid receptor), having a hormonal effect. A similar sequence of events could have happened in the case of vitamin $\mathrm{D}$ with the important difference that cholecalciferol (vitamin D3) has been synthesized already (as synthesized today) by the effect of solarization, which gradually reduced by the absence of sunlight, as humans migrated to the north. In this case the utilizatory system was already available, as vitamin D3 (cholecalciferol) synthesis was present in the organism before [9]. However, the endogeneous production stopped or decreased, as the inducer (sunlight) was absent. So vitamin $\mathrm{D}$ had to be imported from outside or decreased. In the case of retinol (vitamin A), a similar system must have been developed by the human body. as the specific receptor, as well as the mechanisms of transport, storage and detoxification was absent. It seems likely that the other two lipid soluble vitamins, vitamin E and K [10-12] also had a similar route during the transformation from endocrine disruptors to outer (exo)- hormones. These vitamin-hormones are bound presently by the pregnane-X receptors and the specific prospective receptor (likely) develops later. The phytoestrogens presently are bound by members of steroid receptor superfamily and are waiting for their specific receptor. After having this, they become to the full-value member of the exohormone system.

Vitamin A has a key role in the regulation of many biological processes, such as potent gene expression modulator during embryogenesis, organogenesis, cell differentiation and apoptosis [13]. It is essential for vision, growth, and differentiation in mammals [14]. Categorizing it as an ED-hormone, sheds a different light on its role and brings up intriguing questions, such as how and why the above mentioned very important processes are entrusted to an ED-hormone, which must be obtained from the environment? Vitamin A is stored in the adipose tissue but its source is outside the body, which necessitates some form of co-operation between the human (animal) being and the environment.

The conversion of ED hormones to physiologically active hormones (i.e. lipid-soluble „vitamins” to hormones) fundamentally altered the human body and the presence (the consumption) of these hormones became essential for human life, without them many life functions could not be imagined. It is not known, why these molecules had been picked by the human body for vital functions. It is clear, however that when vitamin-hormones had been built into the human physiological processes, only very few (almost nil) similar candidates were present in the environment which had the potential to become to hormones. At present, thousands of such materials are produced by the industry and are used in agriculture and industry, and they are found in our food, water, air and everyday objects. If the human organisms have a mechanism for internalizing these chemicals they are at their disposal. In principle it can be imagined that during a relatively short time such new outer (exo) -hormones will appear which have ED-hormonal origin.

\section{Discussion}

The environment was always the most important factor shaping human evolution, enforcing the alteration of morphology or function for accommodation or defense. In the case of ED-hormones a more intimate effect is at work, a direct intervention into the development of reproductive organs and other basic life functions. When ED-hormones act in the fetal period, malformations are caused such as cryptorchidism, hypospadias or micropenis $[15,16]$ i.e. the transformation of the shape of the human organism. When they act perinatally, faulty imprinting develops, resulting in functional teratogenicity [6]with lifelong functional effects. This is a direct intervention, which influences the life of the target. At the same time, these effects are inherited epigenetically, altering the characteristics of further generations, causing evolutionary change. According to Crews and McLachlan „consequent changes in DNA methylation patterns at cpG islands would be persistent and heritable" [17] and observations by my group and also by others support this observation [18-24]. The developmental effect and inheritance at first enforces the human organism to develop mechanisms for neutralizing ED-s or reducing their harmful effects, as without these defense mechanisms there would not be sufficient time to prove their benefits.

It is a long process for an ED-hormone to become an endogeneous component, as many factors are needed for the detoxification, for the transport (carriers), for finding of hemispecific receptors and for the development of specific receptors (just for the ED-hormone) etc. The presently known lipid soluble vitamins are in different phases of this process. Vitamin A has a complete stock of these factors, while vitamin $\mathrm{E}$ and $\mathrm{K}$ are in transitional phase. Vitamin $\mathrm{D}$ (cholecalciferol) is a special case, as it was a real endogenous hormone (with an exogeneous production-inducer) before people leaving the sunlight-full Africa. Hence the ED-hormone- vitamin cholecalciferol does not require the preparation of the new factors, the existing binding places and factors used by the endogeneous calcipherol could be used by it.

At present, ED-hormones are considered harmful agents. However, from the perspective of the human evolution, these substances also can be advantageous, as it is the case of lipid soluble vitamins. It seems likely that these molecules would have been classified also as harmful ones, at the time of their discovery, however later their usefulness became apparent in such situations when the human organism was offended by dangerous external factors. Earlier we gave the example of reduced sunlight with human migration to the north, when exogenous calciferol was needed to supply the role of sunlight-induced endogeneous production of vitamin $\mathrm{D}$, or retinol import for normal vision. One could imagine a situation when phytoestrogens are needed for supplementing the reduced level of estrogen, as early or late onset of puberty would be more advantageous and phytoestrogens could help the execution of the program of it [25-27]. In these cases, genistein will be internalized similarly to lipid-soluble vitamins and the whole machinery for their utilization will be built and it joins to the numerous similar molecules participating in the normal physiology. When at present positive effects of phytoestrogens are compared with their harmful ones, already these possibilities are considered. The phytoestrogen example is obvious as these are natural products however, artificial products (such as benzpyrene, dioxin, or bisphenol) could also be internalized and be advantageously utilized as components of the human organism. However, natural products are (and were) at our disposal continuously for very long time, while in a modern, industrialized environment artificial products come and go. However, there are some products which can be durably and easily introduced into our physiology, such as aromatic hydrocarbons (e.g. benzpyrene), which are only moderately toxic in typical concentrations and their hormone-like effect can be utilized for stimulating or inhibiting processes in the human organism

The consumption of EDs can help the European population to defend itself from a lot of diseases which is demonstrated by the differences in their statistical incidences between the European and Asian populations. For example, breast and prostate cancers are much higher in the United States and European countries, compared with Japan and China [25]. This caused that some EDs are recommended 
in the prevention and treatment of tumorous diseases, etc [26]. At the same time, the so called harmful effects also could be beneficial from an other standpoint: EDs are reducing human (male) fertility [20,29]. Considering the rapid growth of human population, one could discover an evolutionary benefit in such reduction of fertility rates. From this point of view the extensive appearance of EDs could be a feedback of cultural (technical) evolution to the biological one, and the conversion of EDs to hormones is a considerate biological defense, leading to a more sustainable mankind with a reduced population $[26,30]$.

\section{Conclusion}

The Asian population consumes soy foods' (a rich source of phytoestrogens) for thousands of years, however the effect of this hormonal provocation - in contrast to the phytoestrogen-less European populations - was not thoroughly recorded.This period of time had to be enough for the infiltration of this EDs into the human organism, similarly to the infiltration of lipid-soluble vitamins to the whole human population, which consumes carrots, milk, egg, fish or mushroom etc. for supplementing vitamin A and D. However, while the consequences of lipid soluble vitamin deficiencies are known, the consequences of phytoestrogen deficiencies are not registered. The physiological effects of vitamin A and D were concluded first of all from their deficiency. Albeit the signs and effects of phytoestrogen overdose are known, there is insufficient knowledge on the effects of phytoestrogen-deficiency in the Asian population, as phytoestrogens are not recorded as vitamins or physiological hormones. In addition, there are well-known diseases caused by vitamin A or D deficiency however, diseases in connection with phytoestrogen deficiency were not observed (or not yet known). The systematic and thorough observation of possible diseases could be a major argument toward the recognition of endocrine disruptor - EDhormone-conversion process.

\section{Conflicts of interest}

There is no conflicts of interest.

\section{References}

1. Stearns SC, Byars SG, Govindaraju DR, Ewbank D (2010) Measuring selection in contemporary human populations. Nat Rev Genet 11: 611-622. [Crossref]

2. Csaba G (2007) Thoughts on the cultural evolution of man. Developmental imprinting and transgenerational effect. Riv Biol 100: 461-474. [Crossref]

3. McLachlan JA (2001) Environmental signaling: what embryos and evolution teach us about endocrine disrupting chemicals. Endocr Rev 22: 319-341.

4. Csaba G (1980) Phylogeny and ontogeny of hormone receptors: the selection theory of receptor formation and hormonal imprinting. Biol Rev Camb Philos Soc 55: 47-63. [Crossref]

5. Csaba G (2008) Hormonal imprinting: phylogeny, ontogeny, diseases and possible role in present-day human evolution. Cell Biochem Funct 26: 1-10. [Crossref]

6. Csaba G (2016) The faulty perinatal hormonal imprinting as functional teratogen. Curr Pediatr Rev 12: 222.

7. Csaba G (2011) The biological basis and clinical significance of hormonal imprinting, an epigenetic process. Clin Epigenetics 2: 187-196. [Crossref]

8. Csaba G (1984) The present state in the phylogeny and ontogeny of hormone receptors. Horm Metab Res 16: 329-335. [Crossref]
9. Csaba G (2017) Vitamin-caused faulty perinatal hormonal imprinting and its consequences in adult age. Physiol Int 104: 217-225. [Crossref]

10. Csaba G and Inczefi-Gonda A (1998) Neonatal vitamin E treatment induces long-term glucocorticoid receptor changes: an unusual hormonal imprinting effect. Life Sci 63 : 101 .

11. Csaba G, Karabelyos CS (2000) Influence of a single treatment with vitamin E or K (hormonal imprinting) of neonatal rats on the sexual behavior of adults. Acta Physiol Hung 87: 25-30. [Crossref]

12. Sharat A and Gomaa N (1972) Hormonal properties of vitamin E and its synergism with gonadal hormones. Qual Plant Mat Veg 22: 91.

13. Tekes K, Gyenge M, Folyovich A, Csaba G (2009) Influence of neonatal vitamin A or vitamin $\mathrm{D}$ treatment on the concentration of biogenic amines and their metabolites in the adult rat brain. Horm Metab Res 41: 277-280. [Crossref]

14. Iskakova M, Karbyshev M, Piskunov A, Rochette-Egly C (2015) Nuclear and extranuclear effects of vitamin A. Can J Physiol Pharmacol 93: 1065-1075. [Crossref]

15. Sultan C, Paris F, Terouanne B, Balaguer P, Georget V, Poujol N, et al. (2011) Disorders linked to insufficient androgen action in male children. Hum Reprod Update 7: 314.

16. Gaspari L, Paris F, Jandel C, Kalfa N, Orsini M, et al. (2011) Prenatal environmental risk factors for genital malformations in a population of 1442 French male newborns: a nested case-control study. Hum Reprod 26: 3155.

17. Crews D and McLachlan JA (2006) Epigenetics, evolution, endocrine disruption, health, and disease. Endocrinology 147: s4

18. Tekes K, Gyenge M, Hantos M, Csaba G (2009) Transgenerational hormonal imprinting caused by vitamin A and vitamin D treatment of newborn rats. Alterations in the biogenic amine contents of the adult brain. Brain Dev 31: 666-670. [Crossref]

19. Csaba G, Kovacs P, Pallinger E (2007) Transgenerational effect of neonatal vitamin A or D treatment (hormonal imprinting) on the hormone content of rat immune cells. Horm Metab Res 39: 197-201. [Crossref]

20. Csaba G (2017) The Present and Future of Human Sexuality: Impact of Faulty Perinatal Hormonal Imprinting. Sex Med Rev 5: 163-169. [Crossref]

21. Anway MD, Cupp AS, Uzumcu M, Skinner MK (2005) Epigenetic transgenerational actions of endocrine disruptors and male fertility. Science 308: 1466-1469. [Crossref]

22. Holliday R (1987) The inheritance of epigenetic defects. Science 238: 163-170. [Crossref]

23. Holliday R (1998) The possibility of epigenetic transmission of defects induced by teratogens. Mutat Res 422: 203-205. [Crossref]

24. Csaba G (2014) Transgenerational effects of perinatal hormonal imprinting. In: Transgenerational epigenetics. Ed.: T. Tollefsbol, Chapter 19. Academic Press New York. pp.255-272.

25. Sarkar FH, Li Y (2003) Soy isoflavones and cancer prevention. Cancer Invest 21: 744757. [Crossref]

26. Messina M (2016) Soy and health update: Evaluation of the clinical and epidemiological literature. Nutrients 24: E754

27. Wingfield JC (2008) Comparative endocrinology, environment and global change. Gen Comp Endocrinol 157: 207-216. [Crossref]

28. Byars SG, Ewbank D, Govindaraju DR, Stearns SC (2010) Colloquium papers: Natural selection in a contemporary human population. Proc Natl Acad Sci U S A 107 Suppl 1: 1787-1792. [Crossref]

29. Guerrero-Bosagna C, Skinner MK (2014) Environmentally induced epigenetic transgenerational inheritance of male infertility. Curr Opin Genet Dev 26: 79-88. [Crossref]

30. Stearns SC (1976) Life-history tactics: a review of the ideas. Q Rev Biol 51: 3-47. [Crossref]

Copyright: (C2019 Csaba G. This is an open-access article distributed under the terms of the Creative Commons Attribution License, which permits unrestricted use, distribution, and reproduction in any medium, provided the original author and source are credited. 